\title{
Pyogenic osteomyelitis presenting as an acute sterile arthropathy
}

\author{
PHILIP N. PLATT AND IAN D. GRIFFITHS
}

From the University Department of Rheumatology, Royal Victoria Infirmary, Newcastle upon Tyne

SUMMARY We report two cases of osteomyelitis secondary to staphylococcal septicaemia, presenting with large sterile effusions in adjacent joints. In both cases the clinical features were suggestive of septic arthritis. Multiple joints were involved in each patient. Initial radiographs showed no abnormalities.

Metaphyseal and apiphyseal vessels from anastomoses in the adult. Since the epiphysis and adjacent synovium also share a common blood supply, there is an obvious route for spread of infection to the joint from an adjacent focus of osteomyelitis. ${ }^{1}$ However, recent reviews of osteomyelitis have failed to comment on the occurrence of secondary effusions or septic arthritis, ${ }^{2} 3$ although septic arthritis was regarded as a common but late complication of osteomyelitis in preantibiotic series. ${ }^{2}$ The presence of effusions in these cases as a presenting feature appears to be particularly unusual.

\section{Case reports}

CASE 1

A 16-year-old trainee gardener was admitted to hospital with pyrexia $\left(39^{\circ} \mathrm{C}\right)$, rigors, a diffuse erythematous rash over his trunk, and several swollen joints. Two days prior to admission he had been in the Accident and Emergency Department with a two-day history of pain in his shoulder. At that stage his shoulder was noted to be tender with a restricted range of movements. No other abnormalities were detected, and no further action was taken.

At the time of admission his right shoulder, right elbow, and right knee were swollen, painful, and erythematous, with obvious effusions. There was a raised erythematous skin lesion on the right thigh and a diffuse erythematous rash but no evidence of preceding skin infections or other portals of entry.

Blood cultures were taken and the knee, shoulder, and elbow aspirated. The synovial fluid samples were only slightly cloudy, the white cell

Accepted for publication 20 January 1984.

Correspondence to Dr P. N. Platt, Clinical Lecturer, University Department of Rheumatology, Newcastle upon Tyne. counts varying between 7 and $10 \times 10^{9} / 1$. No organisms were seen on direct Gram stain, and subsequent cultures were sterile. Initial radiographs of the affected joints were normal.

The patient was started on benzyl penicillin and flucloxacillin intravenously. Blood cultures showed a heavy growth of Staphylococcus aureus in all cultures. The benzyl penicillin, to which the organism was resistant, was replaced with fucidin.

Clinically the patient improved rapidly on antibiotics except for his right shoulder and elbow, which remained painful and swollen, with recurrent effusions. The effusions were again aspirated, but no organisms were obtained. Two weeks after the onset of the illness radiographs of the right shoulder, elbow, and humerus showed evidence of severe osteomyelitis involving the whole of the humerus (Fig. 1).

Surgical drainage was not performed. The antibiotics were continued for the total of six months, during which time there was a return to full mobility of the shoulder and elbow. Radiological evidence of healing of the osteomyelitic lesions was seen.

\section{CASE 2}

A 17-year-old male was admitted with a four-day history of a painful swollen right knee. Three weeks prior to admission he had complained of pyrexial illness, with a sore throat and cervical lymphadenopathy, which had settled spontaneously.

On examination he was pyrexial $\left(39^{\circ} \mathrm{C}\right)$, his right knee was obviously swollen, warm, red, and painful, with a limited range of movements. The left knee was also slightly swollen and tender. A clinical diagnosis of septic arthritis was made. The right knee was aspirated, and $25 \mathrm{ml}$ of cloudy synovial fluid was obtained. Gram stain was negative. The 


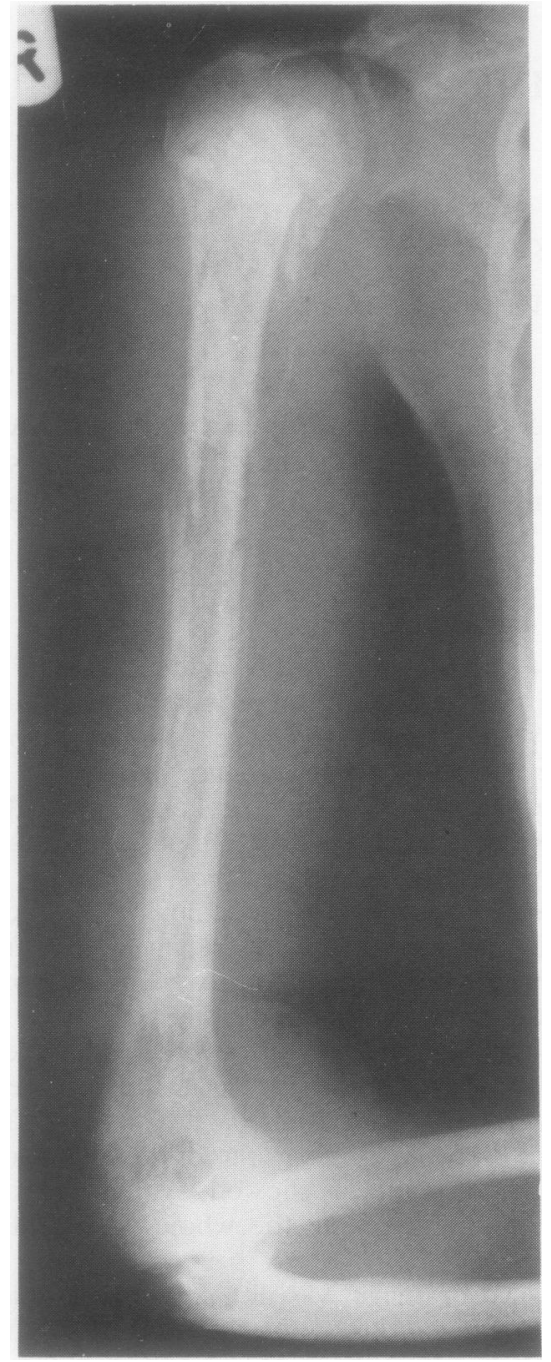

Fig. 1 Radiograph of the right humerus showing extensive changes of osteomyelitis.

total cell count in the synovial fluid was $8 \times 10^{9} / 1$, with $95 \%$ neutrophils. Parenteral antibiotics in the form of ampicillin and flucloxacillin were started. Blood cultures taken at the same time as the knee aspiration showed a moderate growth of Staphylococcus aureus. No organisms were grown from samples of synovial fluid taken from the right knee. Radiographs of the knees on admission were normal.

The knee remained painful, and the effusion recurred. This was aspirated again after three days, with almost identical synovial fluid results. Aspira- tion was again repeated after a further two days, when a small amount of synovial fluid was aspirated with similar characteristics.

Despite treatment the knee remained painful. Repeat radiographs of the knee were performed after 14 days. These showed evidence of a degree of osteopenia of the medial femoral condyle. A technetium-99m methylene disphosphonate bone scan was performed and showed increased uptake in the distal right femoral metaphysis strongly suggestive of osteomyelitis (Fig. 2).

Fucidin was added to the flucloxacillin and antibiotics were continued for a total of four months, by which time the knees had returned to normal with full mobility.

\section{Discussion}

Acute haematogenous osteomyelitis, in which Staphylococcus aureus is the predominant organism, is typically regarded as a disease of childhood. ${ }^{4}$ However, and increasing incidence in older age groups has been reported. ${ }^{2}{ }^{3}$ Trueta $^{1}$ described three types of acute haematogenous osteomyelitis depending on the age of the patient and the changes in vascular anatomy at the corresponding ages. Between the ages of 1 and puberty infection starts in the metaphyseal veins and is contained by the epiphyseal growth plate. In the infant and in the adult there are anastomoses between the metaphyseal and epiphyseal vessels and hence the adjacent synovium, producing an obvious route for the spread of infection to adjacent joints. However, sympathetic effusions and septic arthritis have not been reported as features of osteomyelitis in recent series. $^{23}$ Atcheson and $\mathrm{Ward}^{4}$ describe a patient with acute osteomyelitis progressing to septic synovitis and eventual septic arthritis. They also stress that in this type of case septic synovitis must precede infection of the synovial fluid. This may account for the relatively mild inflammatory changes seen in the synovial fluid of these patients.

Our two cases are atypical in that they presented as an acute arthropathy resembling septic arthritis. Radiological evidence of osteomyelitis was not initially present. In both cases the systemic features and positive blood cultures in addition to the articular findings led to an early and effective antibiotic regimen which may have prevented the progression to frank septic arthritis. However, the age of these patients, 16 and 17 , is such that the adult vascular pattern may not have become fully established, and the possible routes of spread from adjacent osteomyelitis to joint cavity may not have been available. 


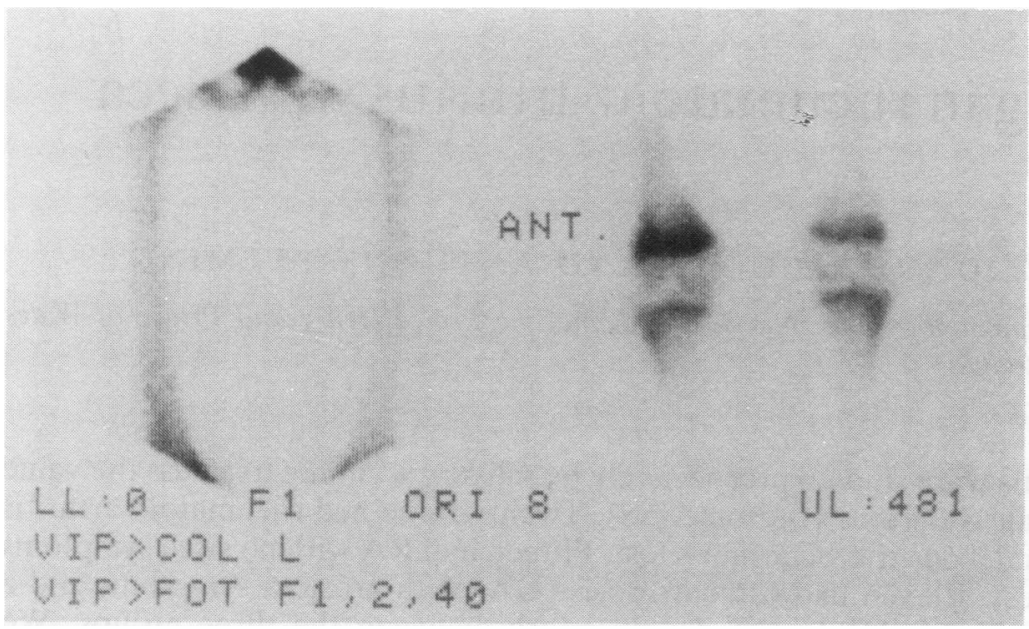

Fig. 2 Technetium-99m methylene diphosphonate bone scan showing evidence of an increased uptake in the right distal femoral metaphysis.
The presence of clinically detectable effusions in joints not adjacent as well as those adjacent to the areas of osteomyelitis suggest that mechanisms apart from secondary spread from osteomyelitis may also have been operating.

\section{References}

1 Trueta J. The three types of acute haematogenous osteo- myelitis: a clinical and vascular study. J Bone Joint Surg 1959; 41B: $617-80$.

2 Waldvogel F A, Medoff G, Swartz M N. Osteomyelitis: a review of clinical features, therapeutic considerations and unusual aspects. $N$ Engl J Med 1970; 282: 198-206.

3 Glover S C, KcKendrick M W, Padfield C, Geddes A M, Dwyer N St J P. Acute osteomyelitis in a district general hospital. Lancet 1982; i: 609-11.

4 Atcheson S G, Ward J R. Acute haematogenous osteomyelitis progressing to septic synovitis and eventual pyarthrosis. Arthritis Rheum 1978; 21: 968-71. 